\title{
Letter to the Editor concerning "Predicting a beneficial response to motor control training in patients with low back pain: a longitudinal cohort study" by Hides JA, et al. (Eur Spine J. 2019; doi.org/10.1007/ s00586-019-06045-7)
}

\author{
Peter Kent ${ }^{1,2}$ (D) Mark Hancock $^{3}$
}

Received: 28 July 2019 / Revised: 28 July 2019 / Accepted: 17 August 2019 / Published online: 24 August 2019

(c) Springer-Verlag GmbH Germany, part of Springer Nature 2019

We read with interest the recent paper titled "Predicting a beneficial response to motor control training in patients with low back pain: a longitudinal cohort study." This study had the commendable aim of identifying patient characteristics that could predict who is likely to benefit most from a physiotherapy intervention, in this context, motor control training (MCT). The study design used data from a large cohort of people, who all received motor control training, to identify which characteristics measured at the first clinical consultation were associated with outcomes (improved or not improved), and to create a predictive model based on those characteristics. The study identified four characteristics and concluded that "... variables which were predictive of a positive response to MCT could be identified at the first treatment session."

Unfortunately, it is not accurate to describe predictors of outcome in a cohort study as predictors of treatment response. That is because, while cohort (single-group) studies of people receiving a particular treatment are useful for identifying prognostic markers, only two-group studies (i.e. a randomized controlled trial) can identify whether they are treatment effect modifiers (identifiers of who is most likely to respond to a particular treatment). At best, such cohort studies can only generate very tentative hypotheses about what prognostic markers might possibly be treatment effect modifiers. The reason is that analysis of cohort-derived data cannot differentiate between (1) a prognostic influence that is unrelated to treatment exposure (the patient would have become better or worse regardless of getting that treatment) and (2) predictors of treatment response (the patient responded to that particular treatment in a way that can be predicted). See key point 1 in Hancock et al. 2009.

It is noteworthy that performing an internal validation does not change this design constraint or assist in differentiating prognostic markers from potential treatment effect modifiers.

In our view, the study by Hides et al. uses language and reaches conclusions that are likely to be misleading, based on the study design used. The precision of language is important, and in our view, the appropriate language in this study would have been to describe the variables as "predictors of outcome" rather than "predictors of treatment response."

Publisher's Note Springer Nature remains neutral with regard to jurisdictional claims in published maps and institutional affiliations.

Peter Kent

peter.kent@curtin.edu.au

1 School of Physiotherapy and Exercise Science, Curtin University, Perth, Australia

2 Center for Muscle and Joint Health, Department of Sports Science and Clinical Biomechanics, University of Southern Denmark, Odense, Denmark

3 Department of Health Professions, Macquarie University, Sydney, Australia 